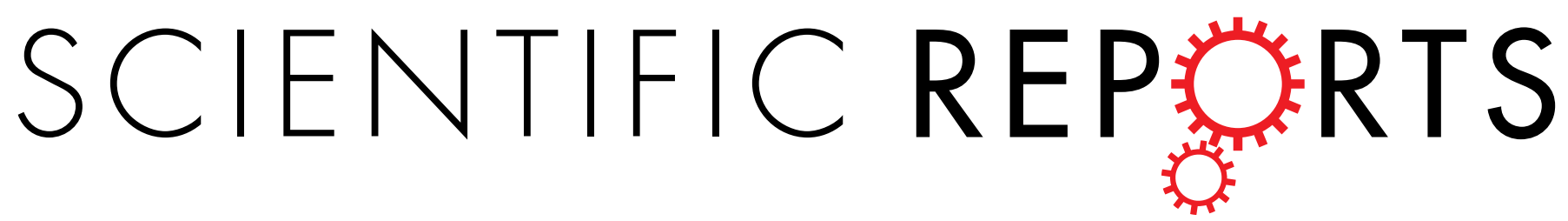

\title{
OPEN Erratum: Advances in mapping malaria for elimination: fine resolution modelling of Plasmodium falciparum incidence
}

Victor A. Alegana, Peter M. Atkinson, Christopher Lourenço, Nick W. Ruktanonchai, Claudio Bosco, Elisabeth zu Erbach-Schoenberg, Bradley Didier, Deepa Pindolia, Arnaud Le Menach, Stark Katokele, Petrina Uusiku \& Andrew J. Tatem

Scientific Reports 6:29628; doi: 10.1038/srep29628; published online 13 July 2016; updated 14 September 2016

In this Article, the columns in Table 2 are misaligned. The correct Table 2 appears below:

\begin{tabular}{|l|c|c|c|c|c|c|}
\hline \multicolumn{2}{|c|}{ Parameter } & Mean & Standard Deviation & $\mathbf{5 \%}$ & Median & $\mathbf{9 5 \%}$ \\
\hline \multirow{2}{*}{ Intercept } & $\mathrm{I}_{\mathrm{D}}$ & -1.0369 & 0.1600 & -1.3004 & -1.0369 & -0.7733 \\
\cline { 2 - 7 } & $\mathrm{I}_{\mathrm{p}}$ & -1.5049 & 0.0620 & -1.9362 & -1.5047 & -1.0738 \\
\hline \multirow{2}{*}{ Precipitation } & $\beta_{\mathrm{P} 1}$ & -0.0025 & 0.0202 & -0.0409 & -0.0025 & 0.0360 \\
\hline EVI & $\beta_{\mathrm{P} 2}$ & -0.0817 & 0.0275 & -0.1271 & -0.0817 & 0.0311 \\
\hline Precision for month & $\tau_{\mathrm{t}}$ & 0.8953 & 0.0908 & 0.2988 & 0.5456 & 3.0182 \\
\hline Precipitation & $\beta_{\mathrm{D} 1}$ & 0.1339 & 0.0145 & 0.1101 & 0.1339 & 0.1578 \\
\hline \multirow{2}{*}{ Spatial range } & $\mathrm{r}_{\mathrm{P}}$ & 0.2470 & 0.0319 & 0.2005 & 0.2435 & 0.3048 \\
\cline { 2 - 7 } & $\mathrm{r}_{\mathrm{D}}$ & 2.1118 & 0.4666 & 1.3440 & 2.1122 & 2.8869 \\
\hline Correlation & $\mathrm{Corr}(\mathrm{P}, \mathrm{D})$ & 0.9353 & 0.0312 & 0.8848 & 0.9344 & 0.9882 \\
\hline Gaussian white noise & $\sigma_{\mathrm{ep}}{ }^{2}$ & 1.4549 & 0.0244 & 1.4150 & 1.4545 & 1.4957 \\
\hline \multirow{2}{*}{ Spatial Variance } & $\mathrm{v}_{\mathrm{p}}$ & 0.3145 & 0.0258 & 0.2750 & 0.3124 & 0.3610 \\
\cline { 2 - 7 } & $\mathrm{v}_{\mathrm{D}}$ & 1.6665 & 0.0081 & 1.6530 & 1.6666 & 1.6799 \\
\hline
\end{tabular}

(i) This work is licensed under a Creative Commons Attribution 4.0 International License. The images or other third party material in this article are included in the article's Creative Commons license, unless indicated otherwise in the credit line; if the material is not included under the Creative Commons license, users will need to obtain permission from the license holder to reproduce the material. To view a copy of this license, visit http://creativecommons.org/licenses/by/4.0/

(c) The Author(s) 2016 